\title{
Computer Simulations for Stability Analysis of a Numerical Procedure for Crossbar Packet Switch
}

\author{
T. Tashev, V. Monov, R. Tasheva
}

Key Words: Computer technologies; simulations; generalized nets; numerical methods; crossbar switch.

\begin{abstract}
The paper is concerned with the problem of calculating the bound of the throughput (THR) of a crossbar packet switch. For this purpose a suitable numerical procedure is used. The input data for the procedure are the results of computer simulations executed on a grid-computational structure (www.hpc.acad.bg). The modeling of the THR uses MiMa-algorithm for a crossbar packet switch, specified by the apparatus of Generalized Nets. For studying the stability of the numerical procedure we use a modified family of patterns for i.i.d. Bernoulli uniform load traffic simulation. The obtained results show that the numerical procedure is stable which means that small input perturbations result in small changes in the output.
\end{abstract}

\section{Introduction}

A crossbar packet switch maximizes the rate of data transfer using parallel tracks between his input and output. In the ideal case the switch node must send packets without delay and without losses. This is obtained by means of a conflict-free schedule calculated by the control block of the switch node (Scheduler - figure 1) [1,2].

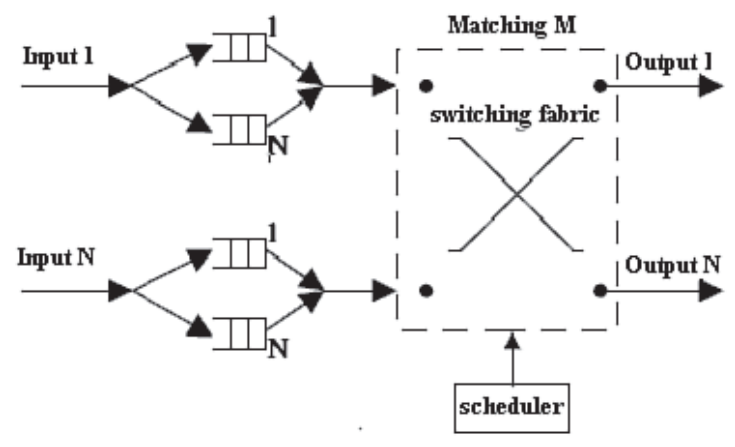

Figure 1. Third generation crossbar switch structure

From a mathematical point of view, the calculation of this schedule is an NP-hard task [3]. The existing approaches partly solve the problem by using different formal apparatus [4]. The observed increase in the capacity of telecommunications traffic requires new algorithms, which have to be examined for efficiency [5]. Similar problems arise in the development of wireless sensor nets [6]. The efficiency of the node is firstly evaluated by the throughput of the switch [4]. In what follows the throughput will be denoted by THR.
At the stage of switch design the THR obtained by different algorithms is initially assessed. The THR will depend on the incoming traffic for a chosen algorithm. When the traffic model is given, the THR depends on the incoming intensity $\boldsymbol{\rho}$ of input lines [4].

In this paper the problem for calculation of the upper bound of the THR is considered. In our computations we use a numerical procedure [7] with input data obtained from high performance calculations. The computer simulation of the THR is executed on the grid-structure of Institute IICTBAS (www.hpc.acad.bg). Our modeling of the THR uses the "Minimum of Maxima" algorithm [8], denoted by MiMa, for switch with commutation field size $(n \times n)$. This algorithm is specified by the apparatus of Generalized Nets (GN) [9]. We will investigate the stability of the numerical procedure with respect to small perturbations in the input (intensity $\boldsymbol{\rho})$ and their effect on the output (THR). For this purpose we use a modified family of patterns [8] for i.i.d. Bernoulli uniform load traffic [10] simulation with an asymptotically vanishing perturbation.

\section{The Problem of Throughput Upper Bound Computation}

For a chosen traffic model, algorithm and load intensity $\boldsymbol{\rho}$, THR depends on the size of its commutation field $n \times n$ ( $n$ input lines, $n$ output lines) and the size $i$ of the input buffer. In practice, the THR is obtained for discrete values of $n(2,3,4,8,16, \ldots)$ and the buffer is presumable to be large. These data are commonly used to estimate the upper bound of THR for $n \rightarrow \infty$ and $i \rightarrow \infty[2,4]$.

In our simulations we examine THR for each integer value in the chosen intervals for $n$ and $i$. The usage of the grid-structure for high performance computations enables us to perform such simulations and as a result, we can obtain a more accurate estimation of the upper bound.

We shall use the following definitions [11] :

Definition 1. Function $\mathrm{f}$ is defined as

$$
\text { (1) } f(n, i)=\operatorname{THR}(n, i)
$$

where $n=2,3, \ldots$ is the size of the input /output lines of the packet node, $i=1,2, \ldots$ is the size of the input buffer of each input line and $1 \geq \operatorname{THR}(\mathrm{n}, \mathrm{i}) \geq 0$.

THR with value one corresponds to the maximal normalized throughput (100\%). 
Definition 2. Function V is defined as

$$
\text { (2) } \begin{aligned}
V(n)=\lim _{i \rightarrow \infty} f(n, i) \\
n \in[a, b]
\end{aligned}
$$

where $i \rightarrow \infty$ means infinitely large input buffer and $n$ belongs to the interval $[\mathrm{a}, \mathrm{b}]=\left[n_{1}, n_{2}\right]$ which is determined by the working range of computer simulations - for size of the input/output lines from $n_{1}$ to $n_{2}$. Typically, $n_{1}$ equals 2 .

Thus, $V(n)$ is the upper bound of the THR within the range of our simulations of the switch node.

Definition 3. The absolute upper bound U of THR is defined as

$$
\text { (3) } U=\lim _{\substack{i \rightarrow \infty \\ n \rightarrow \infty}} f(n, i)
$$

where $\mathrm{i} \rightarrow \infty$ means infinitely large input buffer and $\mathrm{n} \rightarrow \infty$ means infinitely large commutation field.

If $V(n)$ is known, the calculation of the $U$ is a standard task. Therefore we focus on the following Problem.

Problem. Determine $V(n)$ from known results of $f(n$, $i$ ) for THR obtained from computer simulations for $n \in\left[n_{1}\right.$, $\left.n_{2}\right], i \in\left[i_{1}, i_{2}\right]$.

We have solved this problem in two steps: establishing the existence of solution and calculating of the solution. We calculate the difference res, between two successive curves $f_{j}$ and $f_{j+1}$, then calculate the ratio $\delta_{j}$ for difference res $s_{j}$ etc. The description of the numerical procedure for calculating of $V(n)$ is given in [11].

\section{Asymptotically Vanishing Perturbation for Stability Analysis of the Numerical Pocedure}

Our stability analysis is based on introducing small perturbations in the input traffic and estimating their influence on the outgoing throughput. The perturbations are introduced in uniform load traffic as follows. We use the family template for a uniform model Uni ${ }^{i}$ shown in figure 2 [8]. This family has $\boldsymbol{\rho}=100 \%$ load intensity of each input line (i.i.d. Bernoulli). Then, we reduce the number of requests in a chosen (e.g., the first) input line by one, i.e. minus one request. In this case the general element $\mathrm{T}_{\mathrm{p}}^{\mathrm{i}}$ for $(\mathrm{k} \times \mathrm{k})$ size of the pattern $\mathrm{U}_{\mathrm{p}}^{\mathrm{i}}$ for the model with perturbations becomes as it is shown in figure 3. The resulting throughput is the average for $\mathrm{n}$ runs for each size $(n \times n)$.

$$
\begin{aligned}
& T^{1}(2 \times 2)=\left[\begin{array}{cc}
1 & 1 \\
1 & 1
\end{array}\right] ; T^{1}(3 \times 3)=\left[\begin{array}{ccc}
1 & 1 & 1 \\
1 & 1 & 1 \\
1 & 1 & 1
\end{array}\right] ; \ldots ; T^{1}(k x k)=\left[\begin{array}{ccc}
1 & \ldots & 1 \\
\vdots & \ddots & \vdots \\
1 & \ldots & 1
\end{array}\right] ; \ldots \\
& T^{i}(2 \times 2)=\left[\begin{array}{cc}
i & i \\
i & i
\end{array}\right] ; T^{i}(3 \times 3)=\left[\begin{array}{ccc}
i & i & i \\
i & i & i \\
i & i & i
\end{array}\right] ; \ldots ; T^{i}(k x k)=\left[\begin{array}{ccccc}
i & \ldots & i & \ldots & i \\
\vdots & & & \\
i & \ldots & i & \ldots & i \\
\vdots & & & & \vdots \\
i & \ldots & i & \ldots & i
\end{array}\right] ; \ldots
\end{aligned}
$$

Figure 2. The family of patterns for uniform load traffic: Uni ${ }^{1}$ and $\mathrm{Uni}^{\mathrm{i}}$

$$
\begin{aligned}
& T_{p}^{1}(2 \times 2)=\left[\begin{array}{ll}
0 & 1 \\
1 & 1
\end{array}\right],\left[\begin{array}{ll}
1 & 0 \\
1 & 1
\end{array}\right] ; T_{p}^{1}(3 \times 3)=\left[\begin{array}{lll}
0 & 1 & 1 \\
1 & 1 & 1 \\
1 & 1 & 1
\end{array}\right],\left[\begin{array}{lll}
1 & 0 & 1 \\
1 & 1 & 1 \\
1 & 1 & 1
\end{array}\right],\left[\begin{array}{lll}
1 & 1 & 0 \\
1 & 1 & 1 \\
1 & 1 & 1
\end{array}\right] ; \ldots \\
& T_{p}^{i}(3 \times 3)=\left[\begin{array}{ccc}
i-1 & i & i \\
i & i & i \\
i & i & i
\end{array}\right],\left[\begin{array}{ccc}
i & i-1 & i \\
i & i & i \\
i & i & i
\end{array}\right],\left[\begin{array}{ccc}
i & i & i-1 \\
i & i & i \\
i & i & i
\end{array}\right]
\end{aligned}
$$

Figure 3. The part of family of patterns for uniform traffic with pertubations $\mathrm{Up}^{1}$ and $\mathrm{Up}^{\mathrm{i}}$

The family of patterns $U_{p}{ }^{i}$ corresponds to a load intensity with perturbations in the first input line. For this input the load intensity is $\boldsymbol{\rho}=(100-\Theta) \%$ where $\Theta$ is a asymptotically vanishing perturbation. The curves for $\Theta$ and $\rho$ are shown in figure 4 , where $\Theta=(i x n-1) /(i x n)$ is normalized with respect to $100 \%(\boldsymbol{\rho}=1), \mathrm{i}=1,2,4,8,256$.
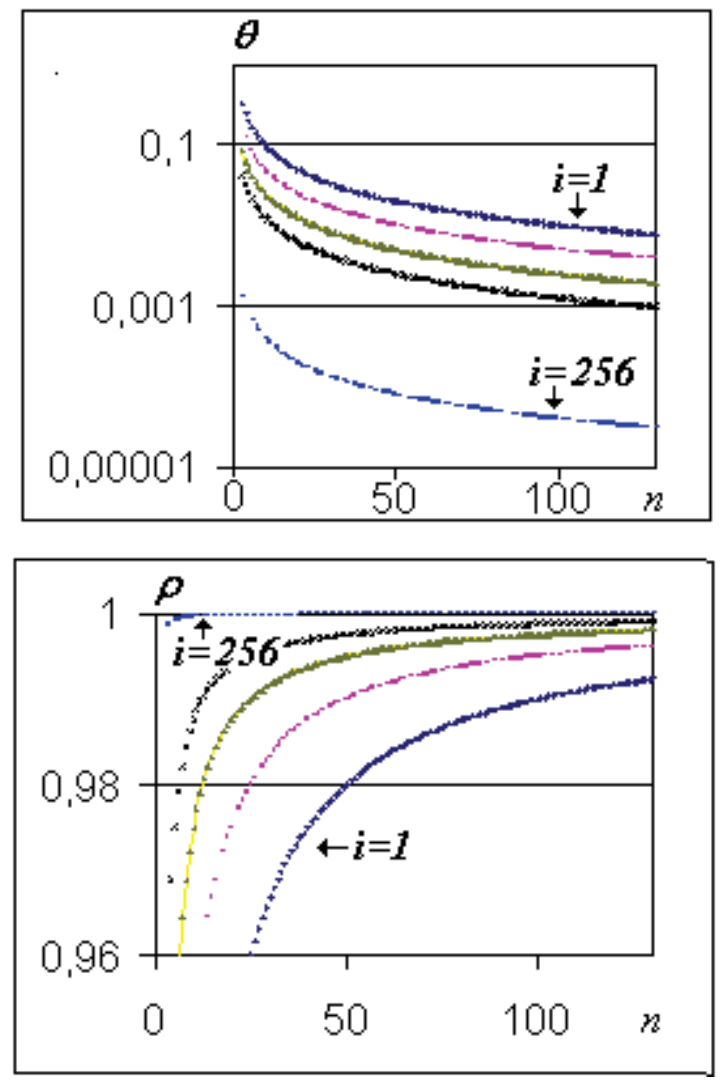

Figure 4. The curves for perturbation $\Theta$ and intensity $\rho$

\section{Computer Simulations of Throughput}

In the computer simulation we are utilizing Generalized-Net-model of the MiMa-algorithm [12]. The transition to executive program from the GN-model is performed as in [13] using the program VFort [14]. The source code has been compiled by means of the grid-structure BG01-IPP of the Institute IICT - Bulgarian Academy of Sciences (www. hpc.acad.bg). The resulting code is realized locally in the 
grid-structure. The operation system is SL release 6.5. We used the grid-resources: 32 threads (up to $16 \mathrm{CPU}$ ), 12GB RAM. The restriction is the time for calculation ( $<72$ hours).

We choose value $\mathrm{m}=2$ for comparison with the results from [11]. When $\mathrm{m}=2$, then $i=\mathrm{m}^{\mathrm{p}}, \mathrm{p}=0,1,2, \ldots$ The initial estimate of the required number of curves for THR is at least 4 starting from Pattern $\mathrm{Up}^{1}$. In these simulations we have obtained nine curves for nine patterns $(p=0,1,2, \ldots, 8)$. We get results for $\mathrm{Up}^{1}, \ldots, \mathrm{Up}^{256}$ and figure 5 shows the particular results for $\mathrm{Up}^{1}, \mathrm{Up}^{2}$. The figure 6 shows the particular results for $\mathrm{Up}^{1}$ and $\mathrm{Up}^{256}$. The size $\mathrm{n}$ varies from $3 \times 3$ to $130 \mathrm{x} 130$. The throughput is the average for $\mathrm{n}$ runs.

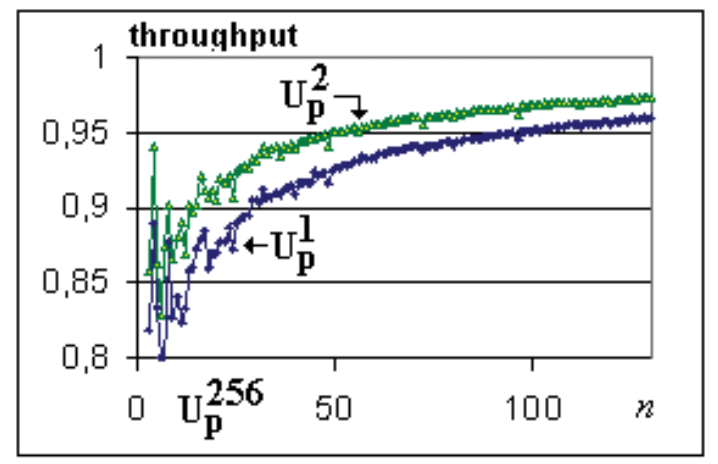

Figure 5. Throughput for uniform traffic with perturbations $\mathrm{Up}^{1}$, $\mathrm{Up}^{2}$

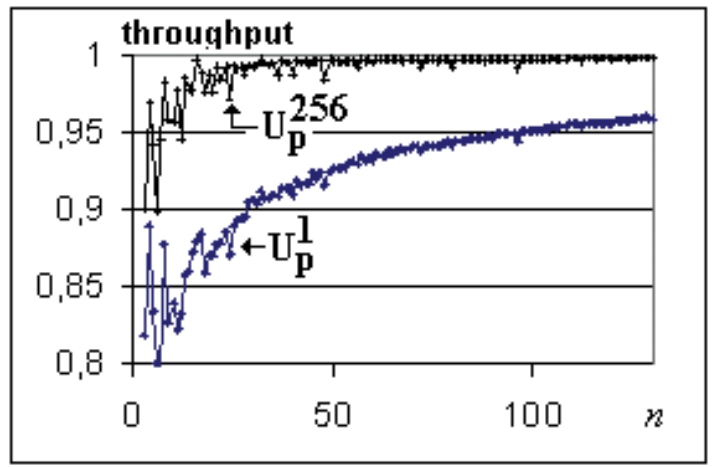

Figure 6. Throughput for uniform traffic with perturbations $U p^{256}$

\section{Assess the Stability of the Numerical Procedure for Upper Bound}

We calculate the ratio $\boldsymbol{\delta}_{\mathrm{j}}$ for differences res $\mathrm{s}_{\mathrm{j}}$ between throughput for neighboring patterns $[7,11]$ and the obtained curves for $\boldsymbol{\delta}_{j}$ are shown in figure 7 (for $j=1$ ) and figure 8 (for $j=7$ ). The values of $\boldsymbol{\delta}_{1}$ are varying above $(1,5)^{-1}$, and the values of $\boldsymbol{\delta}_{7}$ tend to $(1,4)^{-1}=\mathrm{m}^{-1 / 2}$, which is the expected value without perturbation [11].

The upper boundary in case $\mathrm{m}=2$ can be calculated according to [11] as

$$
\begin{aligned}
(4) \mathrm{V}(n)= & \mathrm{f}\left(n, \mathrm{~m}^{(\mathrm{p}-1)}\right)+\left[\delta(\mathrm{m})+\delta^{2}(\mathrm{~m})+\ldots+\right. \\
& \left.+\delta^{\mathrm{p}}(\mathrm{m})+\ldots\right] \cdot \operatorname{res}\left(\mathrm{m}^{(\mathrm{p}-2)}\right) \\
= & \mathrm{f}\left(n, \mathrm{~m}^{(\mathrm{p}-1)}\right)+\left[\left(\mathrm{m}^{1 / 2}-1\right)^{-1}\right] . \\
& \left(\mathrm{f}\left(n, \mathrm{~m}^{(\mathrm{p}-1)}\right)-\mathrm{f}\left(n, \mathrm{~m}^{(\mathrm{p}-2)}\right)\right) .
\end{aligned}
$$

We calculate the boundary for the last simulation $\mathrm{f}(\mathrm{n}, 256)$ (for $\mathrm{p}=8)$ as

$$
\begin{aligned}
& \qquad(n)= \\
& \quad \mathrm{f}(n, 256)+\left[\left(2^{1 / 2}-1\right)^{-1}\right] .(\mathrm{f}(n, 256) \\
& \mathrm{f}(n, 128)) .
\end{aligned}
$$

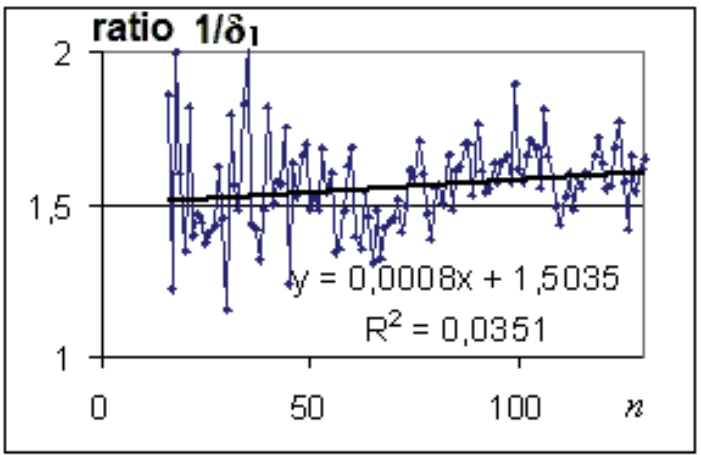

Figure 7. Ratio $1 / \delta_{1}\left(\right.$ for $\left.\mathrm{Up}^{1}, \mathrm{Up}^{2}, \mathrm{Up}^{4}\right)$

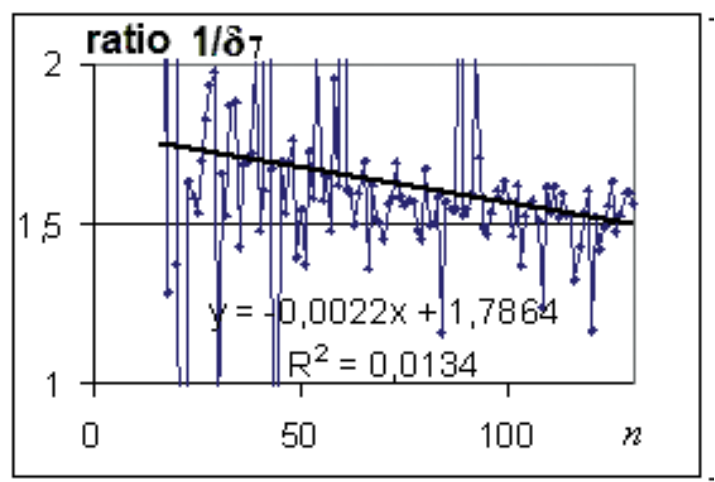

Figure 8. Ratio $1 / \delta_{7}$ (for $\mathrm{Up}^{64}, \mathrm{Up}^{128}, \mathrm{Up}^{256}$ )

This result is shown in figure 9 and it is obtained for the difference $\delta_{7}$ which is most closely tending to $\mathrm{m}^{-1 / 2}$.

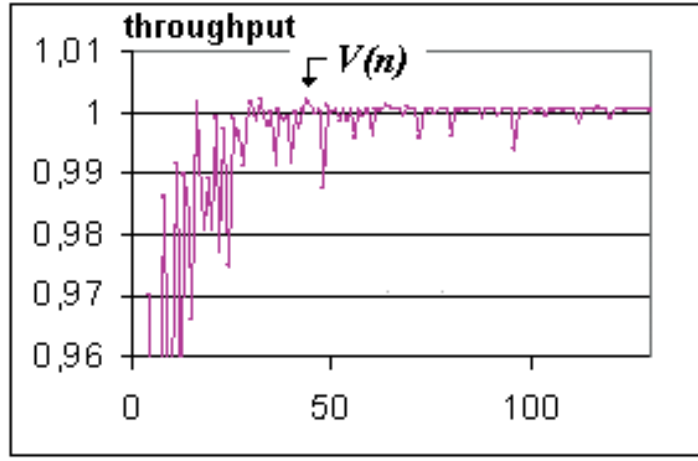

Figure 9. Upper boundary $V(n)$ for $\delta_{7}\left(\right.$ for $\mathrm{Up}^{256}$ )

For comparison, we have calculated the boundary for difference $\delta_{1}$ as

$$
\begin{aligned}
& \mathrm{f}_{i \rightarrow \infty}(n, i)=\mathrm{f}(n, 4)+\left[\left(2^{1 / 2}-1\right)^{-1}\right] \cdot(\mathrm{f}(n, 4)- \\
& \mathrm{f}(n, 2)) .
\end{aligned}
$$

The corresponding curve is shown in figure 10. The results indicate that in the case of asymptotically vanishing perturbations in the input load intensity, the normalized out- 
going throughput tends to 1 . In [8] it is shown that the upper bound of throughput of the MiMa-algorithm for a uniform traffic model is also 1 . Thus, with this type of input perturbations the throughput of the switch tends to its upper bound without perturbation. Hence, we conclude that the numerical procedure is stable under the described conditions.

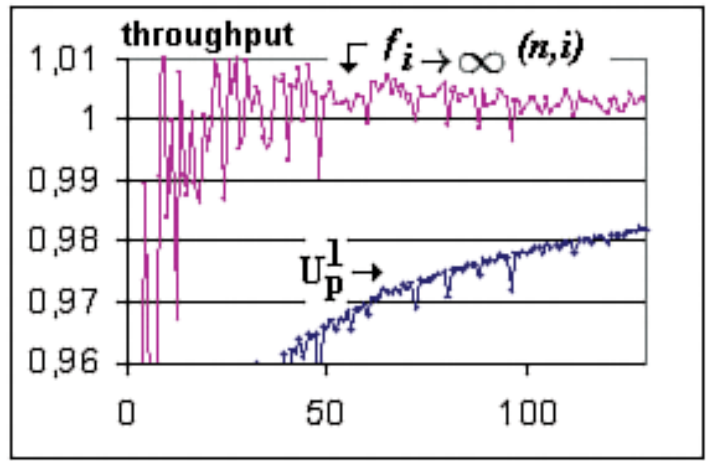

Figure 10. Upper boundary $V(n)$ for $\delta_{1}\left(\right.$ from $\left.\mathrm{Up}^{4}\right)$

\section{References}

1. Deb, S., D. Shah, S. Shakkottai. Fast Matching Algorithms for Repetitive Optimization: An Application to Switch Scheduling. Information Sciences and Systems. 40th Ann. Conference, 22-24 March 2006, 1266-1271.

2. Kang, K., K. Park, L. Sha, Q. Wang. Design of a Crossbar VOQ Real-time Switch with Clock-driven Scheduling for a Guaranteed Delay Bound. - Real-time Systems, 49, 2013, No. 1, 117-135.

3. Chen, T., J. Mavor, Ph. Denyer, D. Renshaw. Traffic Routing Algorithm for Serial Superchip System Customisation. - IEE Proc., 137, 1990, No. 1, 65-73.

4. Chao, H., B. Lui. High Performance Switches and Routers. John Wiley \& Sons, 2007.

5. Staykov, B. Solving Multicriteria Optimization Problems with WebOptim Software System. - Cybernetics and Information Technologies, 15, 2015, No. 3, 165-177.

6. Alexandrov, A. Methods for Optimization of ZigBee Based Autonomous Sensor Systems. Proc. of the International Conference "Automatics and Informatics'2014", 1-3 October 2014, I-183-I-186, Sofia, Bulgaria.

7. Tashev, T., A. Bakanov, R. Tasheva. Determination of the Value of Convergence Parameter in a Procedure of Calculating the Upper Boundary of Throughput for Packet Switch. Proc. of Int. Conference RAM 2013, 8-10 October 2013. Bankya, Bulgaria. Sofia, Prof. M. Drinov Academic Publishing House, 34-37.

\section{Conclusions}

In this work we studied the stability property of a numerical procedure for calculating the upper bound of the throughput of a crossbar packet switch. The result of our computer simulations shows that the numerical procedure is stable in the sense that small values of intensity perturbations of the input traffic lead to small changes in the outgoing throughput of the switch. Also, our simulations indicate that the absolute upper bound of the throughput of the MiMa-algorithm for uniform traffic model with perturbations is $100 \%$.

In a future study, the numerical procedure should be investigated by applying other models of the load traffic in the presence of input perturbations.
8. Tashev, T., T. Atanasova. Computer Simulation of MiMa Algorithm for Input Buffered Crossbar Switch. - International Journal "Information Technologies \& Knowledge", 5, 2011, No. 2, 183189.

9. Atanassov, K. Generalized Nets and System Theory. Sofia, Bulgaria, Prof. M. Drinov Academic Publishing House, 1997.

10. Anderson, T., S. Owicki, J. Saxe and C. Thacker. High Speed Switch Scheduling for Local Area Networks. - ACM Transaction Computer Systems, 11, 1993, No. 4, 319-352.

11. Tashev, T., V. Monov. A Numerical Study of the Upper Bound of the Throughput of a Crossbar Switch Utilizing MiMa-Algorithm. Numerical Methods and Applications (Editors: Ivan Dimov, Stefka Fidanova, Ivan Lirkov), Lecture Notes in Computer Science, 8962, Springer Int. Publishing, 2015, 295-303.

12. Tashev, T. MiMa Algorithm Throughput Modelling for Crossbar Switch with Hotspot Load Traffic. Proc. of the International Conference DCCN'2013, 7-10 October 2013, Moscow, Russia. Moscow, JSC Technosphera, 2013, 257-264 (in Russian).

13. Tashev, T. Modelling Throughput Crossbar Switch Node with Nonuniform Load Traffic. Proc. of the International Conference DCCN'2011, 26-28 October 2011, Moscow, Russia. Moscow, R\&D Company INT, 2011, 96-102 (in Russian).

14. Vabishchevich, P. VFort. http://www.nomoz. org/site/629615/ vfort.html (last checked August 01, 2017). 
Manuscript received on 03.08.2017

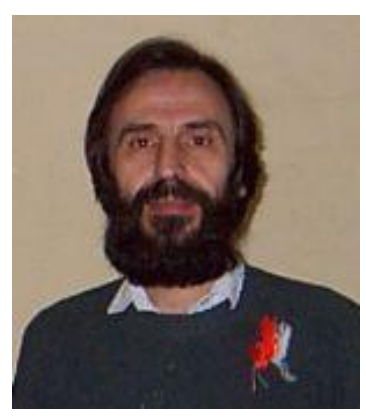

Tasho Tashev graduates the Leningrad Institute of Cinema Engineering (now St. Petersburg Institute of Cinema and TV, Russia) in 1984 as a Radioelectronics Engineer. He received M.Sc. degree in Applied Mathematics from Center of Applied Mathematics, Technical University - Sofia, Bulgaria. The current position is assistant professor in Institute of Information and Communication Technologies - Bulgarian Academy of Sciences. His field of interests includes mathematical modeling, distributed information systems design, methods and tools for network models.

\section{Contacts:}

Institute of Information and Communication Technologies Bulgarian Academy of Sciences Acad. G. Bonchev St., bl. 2, Sofia, Bulgaria e-mail:ttashev@iit.bas.bg

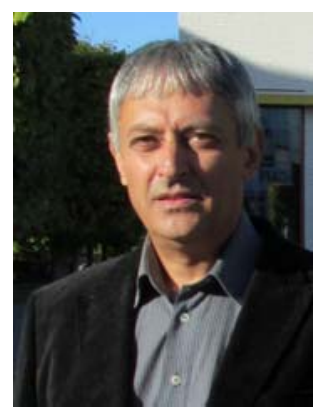

Assoc. Prof. Dr. Vladimir Monov received his M.Sc. degree in Electrical Engineering with qualification in Control Engineering and Automation from Technical University, Sofia and Ph.D. degree in Technical Sciences, Bulgarian Academy of Sciences. Since 2010, Dr. Vladimir Monov has been the Head of Modeling and Optimization Department at the Institute of Information and Communication Technologies, Bulgarian Academy of Sciences. His professional interests and research activity are in the areas of systems and control theory, information and communication systems, business management systems, applied mathematics and operations research.

Contacts: Institute of Information and Communication Technologies Bulgarian Academy of Sciences Acad. G. Bonchev St., bl. 2, Sofia, Bulgaria e-mail:vmonov@iit.bas.bg

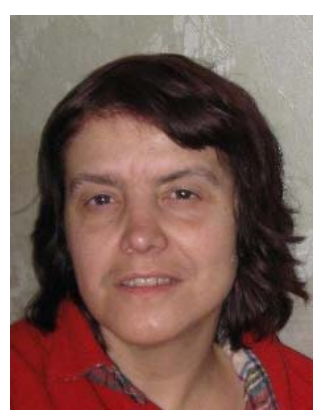

Radostina Tasheva graduates the Sofia University, Bulgaria in 1984 as a Physicist. She received Ph.D. degree in Astronomy from Department of Astronomy, Faculty of Physics, Sofia University in 1991. From 1997 she is assistant professor in Department of Applied Physics, Technical University of Sofia, Bulgaria. Her field of interests includes processes of star formation, galaxies with active nuclei, computer modeling.

Contacts:

Department of Applied Physics

Technical University of Sofia

8 Kliment Ohridski Blvd., bl. 10

1000 Sofia, Bulgaria

e-mail:rpt@tu-sofia.bg 